\title{
Comparing Field Emission Electron Microprobe to Traditional EPMA for Analysis of Metallurgical Specimens
}

\author{
D.F. Susan, R.P. Grant, J.M. Rodelas, J.R. Michael, and M.C. Maguire \\ *Sandia National Laboratories, Albuquerque, New Mexico
}

The use of a thermal field emission electron source and other design changes have significantly increased the resolution of electron probe microanalysis (EPMA), especially in the 5-8kV range of accelerating voltage.[1,2] The analysis of particles/phases as small as $200 \mathrm{~nm}$ in diameter has been documented for geological and meteoritical specimens and a similar analytical volume was shown in a Sn-Ag solder alloy.[1,2] The following work highlights other examples of field emission EPMA for the analysis of fine-scale metallurgical microstructures. Features too small for traditional EPMA, such as fine-scale lamellar transformations and microsegregation in solidification microstructures, can now be analyzed successfully with FE-EPMA. The high resolution is obtained while simultaneously covering a long linescan length or a relatively large area for mapping.

Figure 1 displays a two-phase lamellar structure formed in a multi-component precious metal alloy. The individual layers range from sub-micron to about a micron in width. A traditional EPMA line scan of six elements is shown in the left plot. A typical $15 \mathrm{kV}$ accelerating voltage was employed for this trace with one-micron spacing of analysis points. With conventional microprobe, the lamellar phase compositions can just barely be discerned; silver-rich and copper-rich phases are alternating in the microstructure. In some places only one analysis point is contained within a phase and there is likely significant overlap of analyzed volume from the surrounding phases. The Pd concentration appears relatively flat and the minor constituents of $\mathrm{Au}, \mathrm{Pt}$, and $\mathrm{Zn}$ show slight fluctuations in each phase but it is difficult to quantify the compositional changes. In the right-hand plot, an FE-EPMA line scan of similar length is exhibited, obtained on a JEOL JXA-8530F at 7kV with 0.1 micron spacing. The lamellar phase compositions are now easily determined and the segregation of the minor elements between the two phases is clearly evident as well. The data can be used for phase diagram analysis and to characterize the lamellar discontinuous precipitation mechanism.[3]

In Fig. 2, FE-EPMA analysis of an austenitic stainless steel weld structure is presented. As shown in the elemental map of Ni, the FE-EPMA is able to discern fine scale intercellular segregation of Ni in the weld structure. In the linescan plot, the FE-EPMA results are compared to a traditional EPMA trace. Although the traditional EPMA trace is able to show the general segregation pattern, it does not accurately determine the peaks in Ni concentration. Again, only a few data points are collected in the peaks and valleys of composition. Note the two traces were taken in slightly different locations so the interdendritic patterns do not exactly align spatially. These examples show that FEEPMA can be a powerful analytical tool for studies of solidification/casting and welding as well as many other metallurgical processes producing fine scale compositional fluctuations.

[1] P. McSwiggan et al., Micros. Microanal. 17 (Suppl 2), 2011, 624-625.

[2] J.T. Armstrong et al., Micros. and Analysis, 27(7), Nov. 2013, 17-20.

[3] D.F. Susan et al., Met. Mat. Trans. A, 45(9), Aug. 2014, 3755-3766.

* Sandia National Laboratories is a multi-program laboratory managed and operated by Sandia Corporation, a wholly owned subsidiary Lockheed Martin Corporation, for the U.S. Department of Energy's National Nuclear Security Administration under contract DE-AC04-94AL85000. 

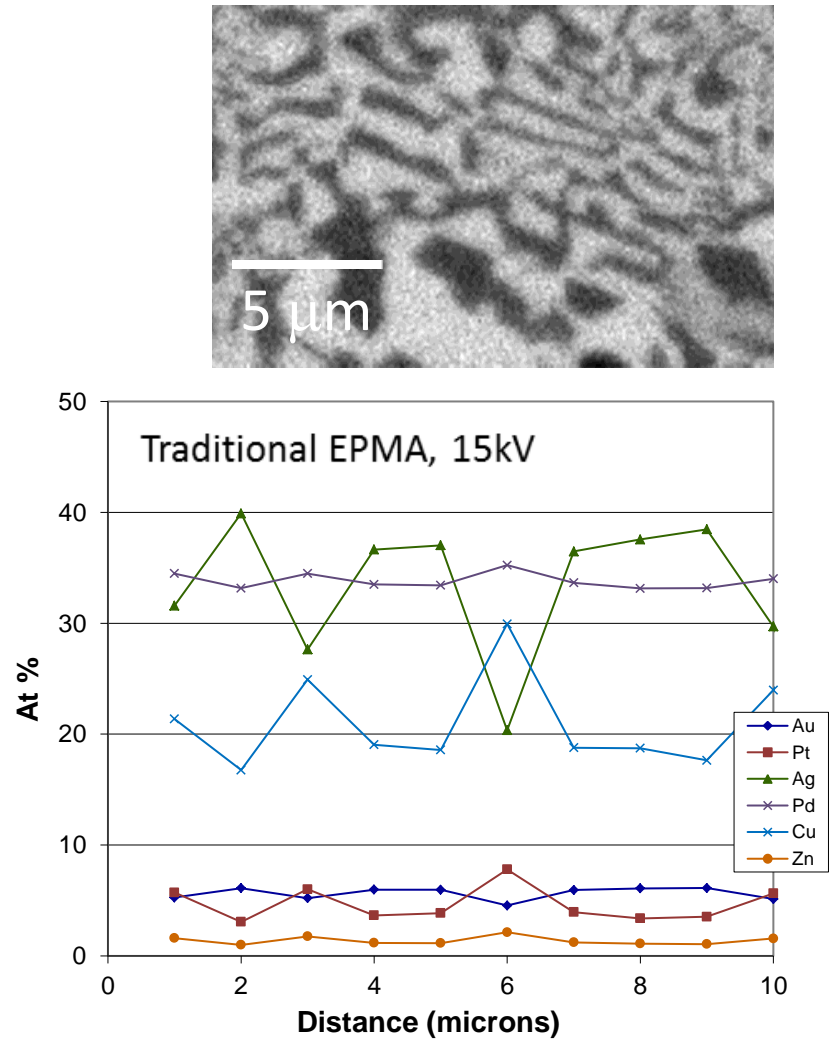
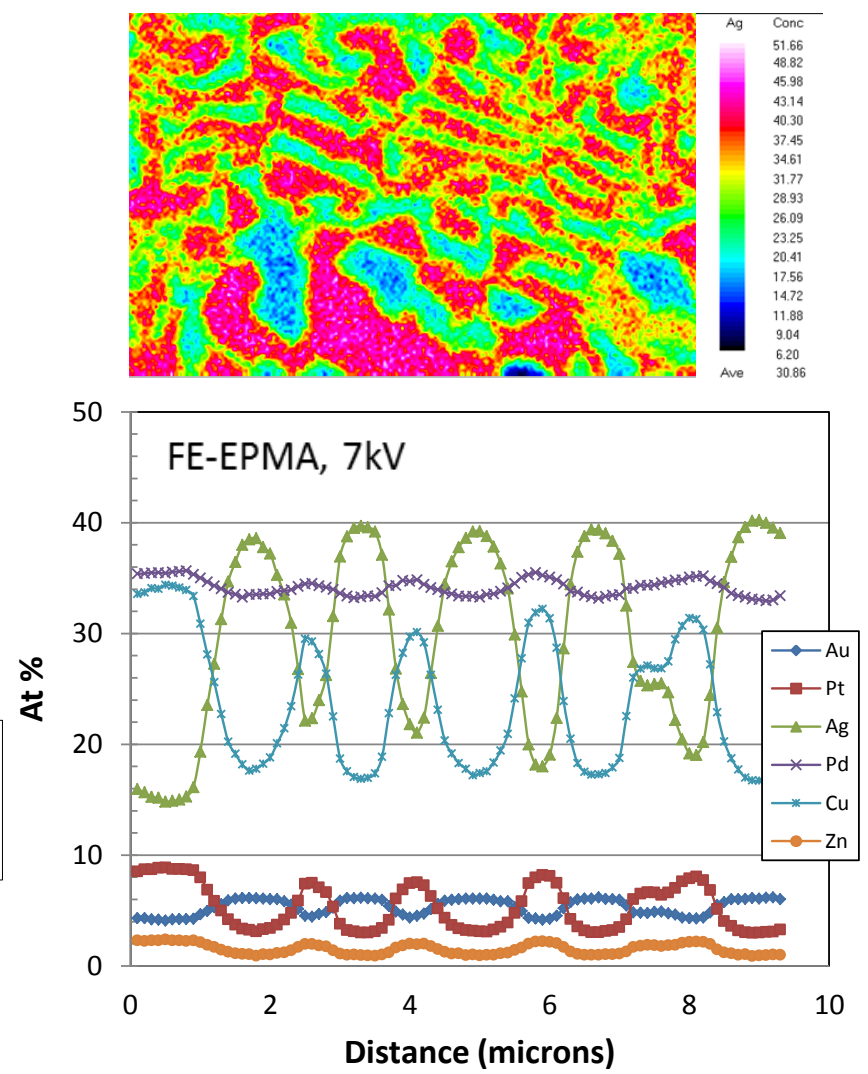

Fig. 1. (top) FE-EPMA elemental maps showing concentration of Ag obtained from a multicomponent precious metal alloy. (bottom) EPMA linescan through the lamellar structure obtained with a traditional EPMA instrument and an FE-EPMA linescan at 7kV on a JEOL JXA-8530F.
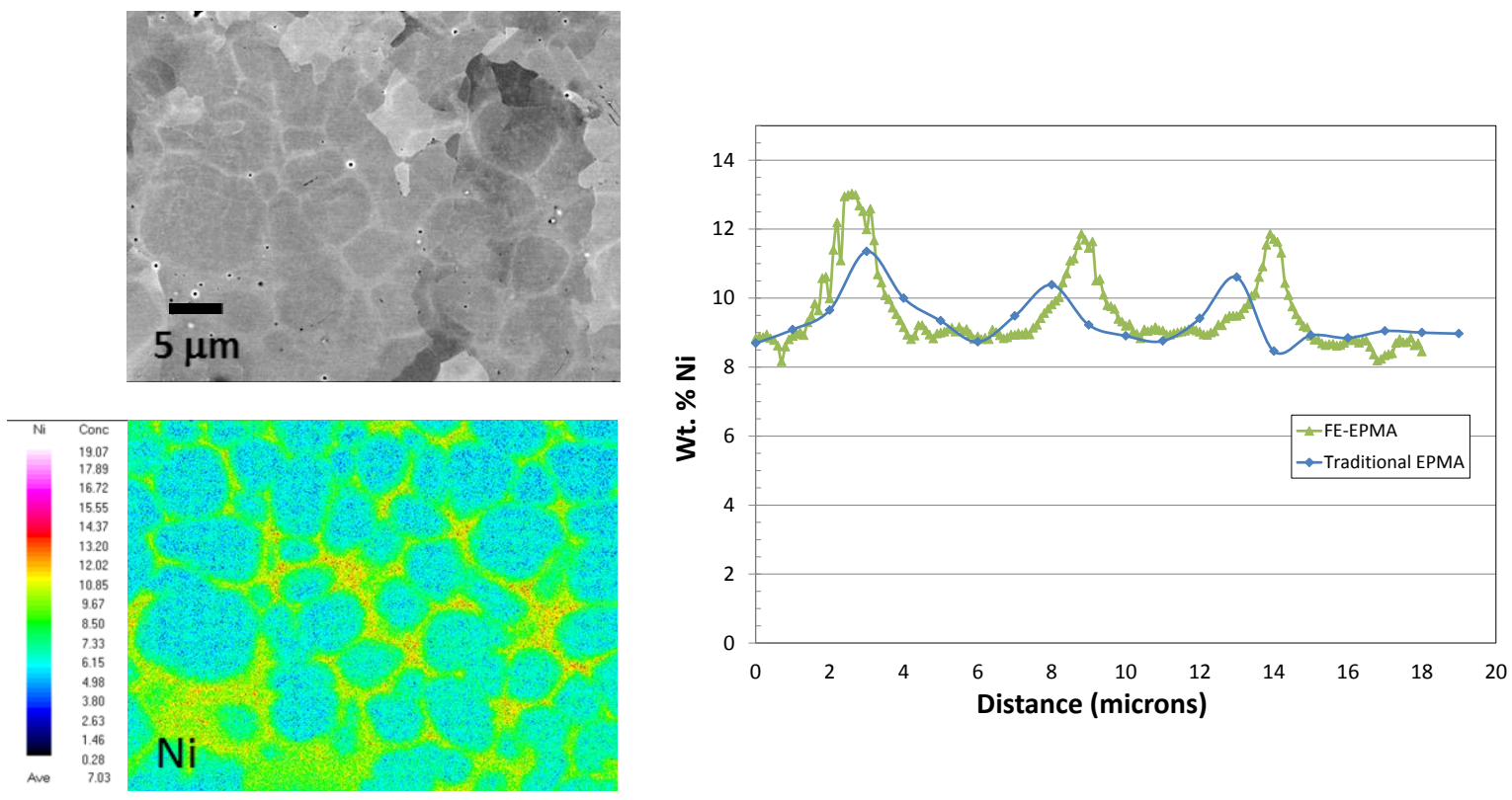

Fig. 2. (left) BSE photo and Ni elemental map from a stainless steel weld microstructure. (right) Traditional EPMA and FE-EPMA linescans for Ni. 